\title{
Evaluation of various active surveillance protocols in prostate cancer
}

\author{
Kayhan Yılmaz ${ }^{1}$, Tahir Karadeniz ${ }^{2}$, Orkunt Özkaptan ${ }^{3}$, Oğuz Yilanoglu ${ }^{4}$ \\ ${ }^{1}$ Antalya Korkuteli State Hospital, Turkey; \\ 2 Medicana Hospital Group Istanbul, Turkey; \\ ${ }^{3}$ Kastamonu State Hospital; \\ ${ }^{4}$ Hatay Dörtyol State Hospital, Turkey.
}

\begin{abstract}
Summary Objective: This study aims to investigate whether pathology results obtained by radical retropubic prostatectomy (RRP) were correlated with active surveillance (AS) criteria defined by Klotz, Soloway and D'Amico.

Materials and Methods: In our clinic we evaluated 211 patients with diagnosis of localized prostate cancer who underwent RRP between 2007 and 2012. AS criteria defined by Soloway ( $c T \leq T 2$, PSA $\leq 15 \mathrm{ng} / \mathrm{dl}$, Gleason $\leq 6)$, Klotz (cTlc-T2a; if age $\geq 70 \mathrm{PSA} \leq 15 \mathrm{ng} / \mathrm{dl}$, if age $<70 \mathrm{PSA} \leq 10 \mathrm{ng} / \mathrm{dl}$; if age $\geq 70$ Gleason $\leq 7(3+4)$, if age $<70$ Gleason $\leq 6)$ and D'Amico (cT1c-T2a, PSA $\leq 10$ $n g / d l$, Gleason $\leq 6$ ) were used in our study. Pathological stages and Gleason scores were evaluated with coherence to AS protocols, mis-staging rates, biochemical recurrence $(B C)$ of the mis-staged patients and death due to prostate cancer Data was analyzed using NCSS 2007 \& PASS 2008 Statistical Software (Utah, USA). Chi square test and Mann-Whitney U test were applied for analyzing qualitative data. Significance was determined as $p<0.05$. Results: 137 (64.9\%) patients were coherent with Soloway AS criteria, 118 (55.9\%) with Klotz AS criteria and 108 (51.1\%) with D'Amico AS criteria.

Histopathological results of the patients grouped according to Soloway, Klotz and D'Amico AS protocols showed high stage prostate cancer in 40 (29.2\%), 32 (27\%) and $27(24.9 \%)$ patients, respectively. High grade prostate cancer rates in Soloway, Klotz, D'Amico groups were 55 (40.2\%), 46 (38\%) and 39 (36.1\%); respectively.

Misstaging rates of Soloway, Klotz and D'Amico AS protocols were determined as 65 (47.4\%), 54 (45.5\%) and 46 (42.5\%), respectively. In the Soloway group BC rate was $21.9 \%$ in those with high stages. Relation between $B C$ and high stage was found to be statistically significant $(p<0.05)$.

Conclusion: Misstaging rates were relatively high in the three groups and there was no difference between the three groups in BC rates. Randomized studies with adequate follow up are needed.
\end{abstract}

KEY WORDS: Active surveillance; Prostate cancer; Radical prostatectomy.

Submitted 23 August 2013; Accepted 5 October 2013

\section{INTRODUCTION}

Prostate cancer is a multifaceted disease in which genetic and environmental factors play an important role. Studies show that prostate cancer is the most common cancer in man over 50 years age and is shown to be the second most reason for death due to cancer (1-3).

Currently prostate cancer has various treatment options according to the stage and clinical course of the disease such as radical retropubic prostatectomy (RRP), brachytherapy, external radiotherapy, hormone therapy and chemotherapy. Although there has been an increase in the early diagnosis and treatment rates in prostate cancer, there has been no significant decrease in mortality. This fact gives rise to the thought that clinically insignificant disease is being treated excessively and active follow up of these patients should be preferred instead of radical treatment. Active surveillance which was first described by Coo et al. $(4,5)$ aims to postpone radical treatment and prevent redundant early treatment.

Active surveillance in prostate cancer has become popular in the last decade $(6,7)$. Patients who are adequate for active surveillance are determined with criteria; appropriate prostate specific antigen (PSA) level, clinical stage and Gleason score in the biopsy (8). However, there is no sufficient randomized data available for supporting these criteria.

In our study, we retrospectively investigated patients diagnosed as having localized prostate cancer in which RRP was performed in relation to three different active surveillance criteria as established by Klotz, Soloway and D'Amico. We evaluated whether the pathology results obtained from the RRP specimens were correlated with these three active surveillance criteria.

\section{Materials AND Methods}

We evaluated 211 patients with localized prostate cancer diagnosis in which RRP was performed between 2007 and 2012 in Okmeydanı Training and Research Hospital/ Istanbul. Patients who underwent previous hormonotherapy and/or 5-alfa reductase inhibitor or pelvic radiotherapy were excluded from the study.

Clinical stages were determined using 2002 TMN classifi-

No conflict of interest declared. 
cation. All the RRP operations were performed by the same urologist and all biopsy and RRP specimens were evaluated by the same pathologist. Since positive biopsy core number and tumor percentage were not present in most of the biopsy pathology results, these active surveillance criteria were not included in the study. The active surveillance criteria defined by Soloway (cT $\leq \mathrm{T} 2, \mathrm{PSA} \leq 15 \mathrm{ng} / \mathrm{dl}$, biopsy Gleason score $\leq 6)$, Klotz (Tlc-T2a; if age $\geq 70$ PSA $\leq 15$ $\mathrm{ng} / \mathrm{dl}$, if age $<70 \mathrm{PSA} \leq 10 \mathrm{ng} / \mathrm{dl}$; if age $\geq 70$ biopsy Gleason score $\leq 7(3+4)$, if age $<70$ biopsy Gleason score $\leq 6)$ and D'Amico (clinical stage T1c-T2a, PSA $\leq 10 \mathrm{ng} / \mathrm{dl}$, biopsy Gleason score $\leq 6$ ) were used in our study. Patients appropriated for the three active surveillance protocols were determined by retrospectively examination of the preoperative PSA value, clinical stage and biopsy Gleason score (Table 1). Pathological stages and RRP specimen Gleason scores were evaluated with coherence to active surveillance protocols, misstaging rates, biochemical recurrence of the misstaged patients and death due to prostate cancer.

The data of the study there analyzed using NCSS (Number Cruncher Statistical System) 2007 \& PASS 2008 Statistical Software (Utah, USA). In addition to descriptive statistical analyses (mean, standard deviation), chi square test was used for analyzing qualitative data and in order to analyze qualitative data in cases of abnormal distribution Mann-Whitney U test was applied. Significance was determined at the level of $\mathrm{p}<0.05$.

\section{Results}

We evaluated 211 patients who underwent RRP according to total PSA, clinical stage and biopsy Gleason scores as active surveillance criteria. Active surveillance criteria defined by D'amico, Soloway and Klotz were shown in Table 1. 137 (64.9\%) of the patients were coherent with Soloway active surveillance criteria (9), 118 (55.9\%) with Klotz active surveillance criteria (7) and 108 (51.1\%) with D'amico active surveillance (10) criteria (Table 1).

The clinical features found coherent with active surveillance protocols were compared to final pathology results. The pathology results that showed high grade prostate cancer, Gleason score sum $\geq 7$ ) (17), extracapsular extension (ECI), seminal vesicle (SVI) and/or lymph node involvement (LNI) were studied and misstaging rates were determined (Table 2).

The histopathological results of the patients grouped according to Soloway active surveillance protocol showed high stage prostate cancer in 40 patients (29.2\%). ECI, SVI, LNI was observed in 32 (23.3\%), 7 (5.1\%) and 1 (0.7\%) patient, respectively. High grade prostate cancer was observed in 55 (40.2\%) patients. Of the 118 patients classified in the Klotz active surveillance protocol 32 (27\%) had high stage prostate cancer. ECI, SVI and LNI were found in $26(22 \%), 5(4.2 \%)$ and 1 (0.8\%), respec-

Table 1.

Criteria for active surveillance protocols.

\begin{tabular}{|l|c|c|c|c|}
\hline Selection criteria & All patients & Soloway & Klotz & $\begin{array}{c}\text { D'Amico } \\
\text { and Coleman }\end{array}$ \\
\hline Clinical stage & - & $\leq \mathrm{T} 2$ & T1c-T2a & T1c-T2a \\
\hline PSA & - & $\leq 15$ & $\begin{array}{l}\text { If age } \geq 70 \leq 15 \\
\text { If age }<70 \leq 10\end{array}$ & $\leq 10$ \\
\hline Biopsy gleason score & - & $\leq 6$ & $\begin{array}{l}\text { If age } \geq 70 \leq 7(3+4) \\
\text { If age }<70 \leq 6 \leq 6\end{array}$ & $\leq 6$ \\
\hline $\begin{array}{l}\text { Patients coherent } \\
\text { with the criteria }\end{array}$ & $211(\% 100)$ & $137(\% 64,9)$ & $118(\% 55,9)$ & $108(\% 51,1)$ \\
\hline
\end{tabular}

Table 2.

Pathology results and misstaging rates of RRP.

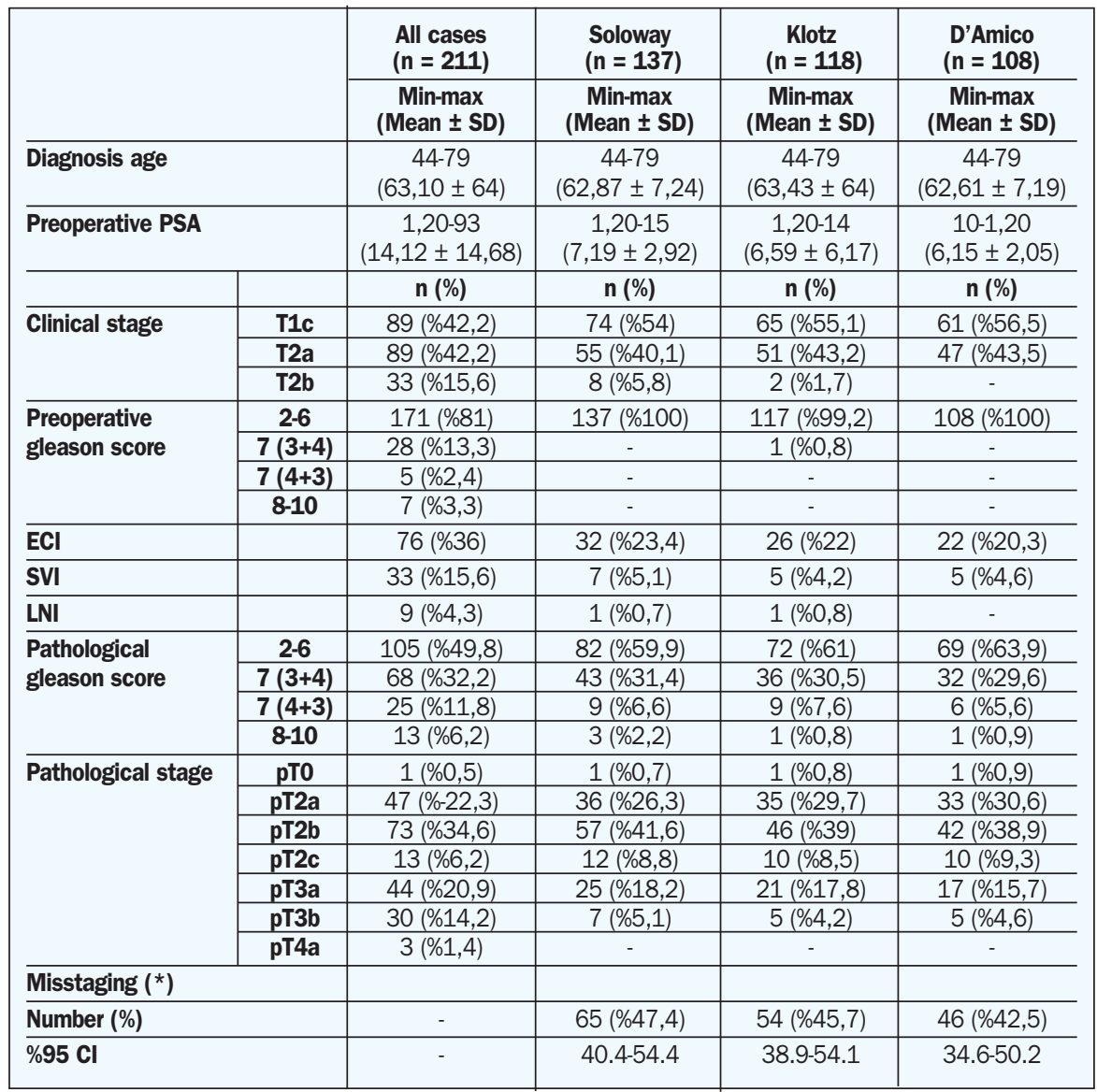

ECI Extracapsular extension. SVI Seminal Vesicle Involvement. LNI Lymph Nod Involvement. 
tively. High grade prostate cancer was present in 46 (38\%) patients. Patients classified according to D'Amico active surveillance criteria comprised 27 (24.9\%) high stage prostate cancer patients. ECI, SVI rates were 22 (20.3\%) and 5 (4.6\%); respectively. In this group 39 (36.1\%) patient were categorized as high grade cancer. Patients diagnosed as organ confined disease on digital rectal examination had local advanced disease in the prostatectomy pathology result with rates of $21.3 \%$ in the group classified by Soloway criteria, $22.8 \%$ in the group according Klotz and 23.3\% in the group according D'Amico. Misstaged patients and misstaging rates of Soloway, Klotz and D'Amico active surveillance protocols were determined as 65 (47.4\%), 54 (45.7\%), 46 (42.5\%); respectively (Table 2 ).

Average follow up periods were 63 months in the Soloway group, 63 months in the Klotz group and 61 months in the D'Amico group. Time until recurrence was 9-48 in the three groups. Total numbers of patients in which biochemical recurrence was detected were $16(11.6 \%)$ in the Soloway group, 13 (11\%), in the Klotz group, 11 (10.2\%) D'Amico group and the period until recurrence in each group was 21, 24 and 25 months, respectively. There was no difference in term of biochemical recurrence rates between the three groups ( $p>0.05)$. In the Soloway group biochemical recurrence rate was obtained to be $21.9 \%$ in those with high stages. The relation between biochemical recurrence and high stage was found to be statistically significant for Solowoy group ( $<<0.05)$ (Tables 3,4$)$. No statistically significant difference was shown between biochemical recurrence rates and high stages according to D'Amico and Klotz criteria $(\mathrm{p}>0.05)$. High Gleason grade ( $\geq 7$ in the prostatectomy specimen) had higher biochemical recurrence values, but this analysis did not reach significance ( $p>0.05)$

There was no statistically significant difference between recurrence and death rates according to misstaging status $(\mathrm{p}>0.05)$. In the Soloway and Klotz groups two deaths due prostate cancer per group and in the D'Amico group one death due prostate cancer were reported.

Deaths due to prostate cancer were only among misstaged patients in the three groups. Statistical analysis was not performed because of the low number of exitus patients (Table 4).
Table 3.

Follow up periods, recurrence and exitus rates distribution in the groups.

\begin{tabular}{|c|c|c|c|c|}
\hline & & Soloway & Klotz & D'Amico \\
\hline \multirow{2}{*}{$\begin{array}{l}\text { Follow up period } \\
\text { (month) }\end{array}$} & Min-max & $13-120$ & $13-118$ & $13-118$ \\
\hline & Mean \pm SD & $63,93 \pm 32,26$ & $63,93 \pm 31,68$ & $61,75 \pm 31,92$ \\
\hline \multirow{3}{*}{ Time until recurrence } & Min-max & $9-48$ & $9-48$ & $9-48$ \\
\hline & Mean \pm SD & $21,87 \pm 12,19$ & $24,17 \pm 12,87$ & $25,10 \pm 13,79$ \\
\hline & & n (\%) & n (\%) & n (\%) \\
\hline \multirow[t]{2}{*}{ Recurrence } & Number (\%) & $16(7,3 \%)$ & $13(11 \%)$ & $11(10.2 \%)$ \\
\hline & $\% 95 \mathrm{Cl}$ & 13.1-19.1 & $10,3-18.9$ & $9,4-18,0$ \\
\hline \multirow[t]{2}{*}{ Death } & Number (\%) & $10(7,3 \%)$ & $8(6,8 \%)$ & $7(6,5 \%)$ \\
\hline & $\% 95 \mathrm{Cl}$ & $3.7-10.9$ & $3.0-10.6$ & $2.6-10.4$ \\
\hline \multirow[t]{2}{*}{ Cause of death } & Prostate cancer & $2(1,4 \%)$ & $2(1,6 \%)$ & $1(0,9 \%)$ \\
\hline & Other & $8(5,9 \%)$ & $6(5,2 \%)$ & $6(5,6 \%)$ \\
\hline
\end{tabular}

Table 4.

Recurrence and death rates according to misstaging status in the groups.

\begin{tabular}{|c|c|c|c|c|}
\hline & & \multicolumn{2}{|c|}{ Misstaging } & \multirow{3}{*}{$\mathbf{P}$} \\
\hline & & Present $\mathbf{n}(\%)$ & Absent n (\%) & \\
\hline \multicolumn{2}{|l|}{ Soloway group } & & & \\
\hline \multicolumn{2}{|l|}{ Recurrence } & $10(\% 15,4)$ & $6(\% 8,3)$ & 0,199 \\
\hline \multicolumn{2}{|l|}{ Death } & $6(\% 9,2)$ & $4(\% 5,6)$ & 0,409 \\
\hline \multirow[t]{2}{*}{ Cause of death } & Prostate & $2(\% 33,3)$ & $0(\% 0)$ & \multirow[t]{2}{*}{-} \\
\hline & Other & $4(\% 66,7)$ & $4(\% 100)$ & \\
\hline \multicolumn{5}{|l|}{ Klotz group } \\
\hline \multicolumn{2}{|l|}{ Recurrence } & $9(\% 16,7)$ & $4(\% 6,3)$ & 0,072 \\
\hline \multicolumn{2}{|l|}{ Death } & $5(\% 9,3)$ & $3(\% 4,7)$ & 0,467 \\
\hline \multirow[t]{2}{*}{ Cause of death } & Prostate & $2(\% 40,0)$ & $0(\% 0)$ & \multirow[t]{2}{*}{-} \\
\hline & Other & $3(\% 60,0)$ & $3(\% 100)$ & \\
\hline \multicolumn{5}{|l|}{ D'Amico group } \\
\hline \multicolumn{2}{|l|}{ Recurrence } & $7(\% 15,2)$ & $4(\% 6,5)$ & 0,136 \\
\hline \multicolumn{2}{|l|}{ Death } & $4(\% 8,7)$ & $3(\% 4,8)$ & 0,456 \\
\hline \multirow[t]{2}{*}{ Cause of death } & Prostate & $1(\% 25,0)$ & $0(\% 0)$ & \multirow[t]{2}{*}{-} \\
\hline & Other & $3(\% 75,0)$ & 3 (\%100) & \\
\hline
\end{tabular}

Chi square test and Fisher's exact was used.

\section{Discussion}

After the description of active surveillance by Coo et a.l, it became more popular for clinicians in the last ten years $(4,6,7)$. Mortality rates of prostate cancer did not decrease despite early diagnosis and treatment of the disease within this period. This fact led clinicians to come to the opinion that clinically insignificant disease is being treated excessively.

Our study was performed to evaluate the credibility of various criteria groups used for the selection of active surveillance patients in order to estimate pathological stage. Misstaging rates of our study for the groups formed according to D'Amico, Klotz and Soloway active surveillance criteria were found to be $42.4 \%, 45.7 \%$ and $47.4 \%$, respectively. With regard to our results D'Amico active surveillance protocol had the most firm patient selection criteria with lower misstaging rates compared to the other groups. In a study by Marc et al., 2837 patients who underwent RRP were evaluated retrospectively.

Patients with appropriate clinical features for separate active surveillance protocols had a misstaging rate between $26-35 \%$ according to their pathological features (11). Nazareno et al. evaluated 4308 patients treated with RRP in five separate active surveillance protocols including D'Amico and Klotz retrospectively. Pathology reports of patients in groups appropri- 
ate for active surveillance were investigated and high stage and/or high Gleason scores were detected in the range $39-56 \%$. According to active surveillance criteria recommended by this study group (PSA $<4 \mathrm{ng} / \mathrm{ml}$, cTl and Gleason score $<7$ ), the misstaging rate $(7.2 \%)$ was found to be statistically significantly decreased. However, the patients' ratio appropriate for active surveillance was found to be decreased to $6.9 \%$ (12) and this rate seemed to be low. In fact in our study the rate of patients suitable for Soloway, Klotz, D'Amico active surveillance protocols were $64.9 \%, 55.9 \%, 51.2 \%$, respectively.

Most important reason for misstaging was determined to be low prostate cancer grade in the biopsy results. The misstaging rate in the biopsy grades with regard to prostatectomy specimen grade were $36.1 \%, 38.9 \%$ and $40.1 \%$, respectively. Supporting our finding, Dall'Era et al. established in their study that the most important reason for changing from active surveillance to radical treatment was the increase in the Gleason grades in prostate biopsies repeated periodically and this ratio was reported to be 38\% (13). In another study performed by Carter et al. this ratio was found to be $30 \%$ (14). In the light of these findings we believe that if tumor grade is detected more accurately at the beginning, approximately $30 \%$ of patients could be treated with active surveillance protocols instead of RRP without losing the chance of cure. Several studies have determined that the sensitivity of digital rectal examination is low in the diagnosis and staging of prostate cancer (15-17). In our study patients with organ confined disease on digital rectal examination had local advanced disease in the prostatectomy pathology results with the rates $21.3 \%, 22.8 \%$, and $23.3 \%$ according to Soloway, Klotz and D'Amico active surveillance groups, respectively. In agreement with this results the evaluation of the propriety of patients for active surveillance showed that there was a misstaging rate of 21.3\%-23.3\% with digital rectal examination and 36.1$40.1 \%$ with Gleason grade. In our study digital rectal examination was found to detect whether the disease is limited to the organ better than grade, however misstaging rates were very high.

The final point in active surveillance is not pathological stage, but biochemical recurrence, metastasis and cancer related death. While radical prostatectomy can cure the disease without affecting the quality of life when performed by experienced surgeons, it is still debated whether one should risk this chance with active surveillance (18). Warlick et al. compared 38 patients who underwent radical prostatectomy following active surveillance with 150 patients with similar characteristics in which radical prostatectomy was performed immediately. This study indicated that postponing prostate cancer surgery didn't risk the chance of cure, however the evident difference between the patient population in the groups decreases the credibility of the study (19). In contrast, the Toronto active surveillance study followed 299 patients and they performed radical prostatectomy in 24 patients which showed progression; in 14 (14\%) pT3 and in two (8\%) N1 was detected and these rates are high (20). This indicated the risk of losing the chance for curative treatment after active surveillance (21-23).

Also the PRIAS (Prostate Cancer Research International:
Active Surveillance) study reported undesired pathological results in 29\% of the patients in which radical surgery was performed following a period of 1.3 years: pT3-4 disease and /or Gleason score $\geq 4+3$ (24). John Hopkins reported that the 10 year disease free rate of $23 \%$ patients in which RRP was performed due to grade progression in the control biopsy was lesser than $75 \%$ (25). However, this rate was not statistically significantly different than patients who have similar clinical features and were operated on with radical surgery within three months (25).

In our study the average follow up periods were 61-63 months in three groups. Biochemical recurrences and rates were $16(11.6 \%)$ in the Soloway group, $13(11 \%)$, in the Klotz group, and 11 (10.2\%) D'Amico group and the period until recurrence in each group was 21,24 and 25 months, respectively. In comparing the three active surveillance groups in our study according to biochemical recurrence, no statistically difference was noted. Statistical significance $(\mathrm{p}<0.05)$ between biochemical recurrence and high stage was remarkable in the Soloway group. Although there was no statistically significant difference in biochemical recurrence rates in high stages according to D'Amico and Klotz criteria, high values of biochemical recurrence in high stage were observed. When evaluating the relationship between Gleason grades in RRP pathologies and biochemical recurrence, patients with high Gleason grades had higher biochemical recurrence values, however this analysis was not statistically significant $(p>0.05)$. In comparing the biochemical recurrence values of misstaged patients and patients who met the criteria in three active surveillance groups, no statistically significant difference ( $p>0.05$ ) was found, however biochemical recurrence rates were higher in misstaged patients. About death due to prostate cancer there were two deaths due to prostate cancer in the Soloway and Klotz group and one death in the D'Amico group. This result was remarkable because all these patients were among the misstaged patients.

\section{ConcLusion}

All these findings show that there are serious problems in the selection of active surveillance patients. Also, there is no consensus in the follow up of active surveillance patient. Consequently the controversial status of active surveillance may result in various mood disorders in patients, and this psychological aspect should not be underestimated. After evaluating all the study results, it is evident that the data on radical surgery results following active surveillance in low risk prostate cancer patients are insufficient. At least one fourth of the pathological data are consistent with the need of treatment and it is unknown how this rate will change with longer surveillance periods and how this will affect the patient prognosis. Thus, there is no current data that postponing active treatment in these patients decreases the chance of cure. Since there are not sufficient randomized studies with adequate follow up periods, active surveillance should only be recommended to a well selected patient group and the patient should be informed about the inconsistencies about active surveillance and all the treatment options should be explained, and the decision should be up to the patient. 


\section{REFERENCES}

1. Sakr WA, Grignon DJ, Crissman JD, et al. High grade prostatic intraepithelial neoplasia (HGPIN) and prostatic adenocarcinoma between the ages of 20-69: an autopsy study of 249 cases. In Vivo. 1994; 8:439-443.

2. Nelson WG, De Marzo AM, Isaacs WB. Mechanisms of the disease: prostate cancer. N Engl J Med. 2003; 349:366-381.

3. Jemal A, Murray T, Ward E, et al. Cancer statistics, 2005; 55:10-30.

4. Choo R, De Boer G, Klotz L, et al. PSA Doubling time of prostate carcinoma managed with watchful observation alone. Int J Radiat Oncol Biol Phys. 2001; 50:615-620.

5. Choo R, Klotz L, Danjoux C, et al. Feasibility study: watchful waiting for localized low to intermediate grade prostate carcinoma with selective delayed intervention based on prostate specific antigen, histological and/or clinical progression. J Urol. 2002; 167:1664-1669.

6. Carter HB, Ferrucci L, Kettermann A, et al. Detection of lifethreatening prostate cancer with prostate-specific antigen velocity during a window of curability. J Natl Cancer Inst. 2006; 98:1521-1527.

7. Klotz L. Active surveillance for prostate cancer: for whom? J Clin Oncol. 2005; 23:8165-8169.

8. Aus G, Abbou CC, Bolla M, et al. EAU guidelines on prostate cancer. Eur Urol. 2005; 48:546-551.

9. Mark S. Soloway, Cynthia T. et al. Active surveillance; a reasonable management alternative for patients with prostate cancer: the Miami experience. B.J.U. International. 2007; 101:165-169.

10. D'Amico AV, Coleman CN. Role of interstitial radiotherapy in the management of clinically organ-confined prostate cancer: the jury is still out. J Clin Oncol. 1996; 14:304-315.

11. Smaldone MC, Cowan JE, Carroll PR, Davies BJ. Eligibility for active surveillance and pathological outcomes for men undergoing radical prostatectomy in a large, community based cohort. J Urol. 2010; 183:138-143.

12. Suardi N, Capitanio U, Chun FK, et al. Currently used criteria for active surveillance in men with low-risk prostate cancer: an analysis of pathologic features. Cancer. 2008; 113:2068-2072.

13. Dall'Era MA., Konety BR, Cowan JE, et al. Active surveillance for the management of prostate cancer in a contemporary cohort. Cancer. 2008; 112:2664-2670.
14. Carter HB, Walsh PC, Landis P, Epstein JI. Expectant management of nonpalpable prostate cancer with curative intent: preliminary results. J Urol. 2002;167:1231-1234.

15. Cevik D, Dillioglugil O. Staging and grading in prostate cancer. In: Ozen H, Turkeri L (eds), Book of Urooncology, Ankara: Ertem Press Publication, 2007; pp. 610-621.

16. Obek C, Louis P, Civantos F, Soloway MS. Comparison of digital rectal examination and biopsy results with the radical prostatectomy specimen. J. Urol. 1999; 161:494-498.

17. Huland H, Hubner D, Henke RP. Systematic biopsies and digital rectal examination to identify nerve-sparing side for radical prostatectomy without risk of positive margin in patients with clinical stage T2, N0 prostatic carcinoma. Urology. 1994; 44:211-214.

18. Klotz L. Active surveillance for prostate cancer: trials and tribulations. World J Urol. 2008; 5:437-444.

19. Warlick C, Trock B, Landis P, et al. Delayed versus immediate surgical intervention and prostate cancer outcome. J Natl Cancer Inst. 2006; 98:355-7.

20. Klotz L. Active surveillance with selective delayed intervention for favorable risk prostate cancer. Urol Oncol. 2006; 24:46-50.

21. Simmons MN, Stephenson AJ, Klein EA. Natural history of biochemical recurrence after radical prostatectomy: risk assessment for secondary therapy. Eur Urol. 2007; 51:1175-1184.

22. Porter CR, Gallina A, Kodama K, et al. Prostate cancer-specific survival in men treated with hormonal therapy after failure of radical prostatectomy. Eur Urol. 2007; 52:446-454.

23. Freedland SJ, Humphreys EB, Mangold LA, et al. Risk of prostate cancer-specific mortality following biochemical recurrence after radical prostatectomy. JAMA 2005; 294:433-439.

24. Bul M, Zhu X, Rannikko A, et al. Radical Prostatectomy for Low-Risk Prostate Cancer Following Initial Active Surveillance: Results From a Prospective Observational Study. Eur Urol. 2012; 62:195-200

25. Soloway MS, Soloway CT, Eldefrawy A, et al. Careful selection and close monitoring of low-risk prostate cancer patients on active surveillance minimizes the need for treatment. Eur Urol. 2010; 58:831-835.

\section{Correspondence}

Kayhan Yllmaz, MD

kayhany@gmail.com

Aşağı Pazar mah. Hastane cad. no 71 Korkuteli Antalya, Turkey

Tahir Karadeniz, MD

karadeniz@gmail.com

Director of Medicana Hospital Group

Beylikdüzü cad. No:3 Beylikdüzü, Istanbul, Turkey

Orkunt Özkaptan, MD

orkunt79@gmail.com

Fellow in Heilbronn Urology Clinic

Tatlipinar caddesi yunus apt no 11 d3 Fatih Istanbul, Turkey

Oğuz Yilanoglu, MD

oyilanoglu@yahoo.com

Yilanoglu, Istasyon Cd. 31600 Dörtyol Hatay, Turkey 